\title{
De nuevo sobre la ortotipografía de las Comedias de Toledo 1500 y Burgos 1499-1502 (?) y Tragicomedias de Zaragoza 1507 y Valencia 1514. Usos gráficos de h, i, y, g, j, x
}

\author{
Remedios Prieto de la Iglesia \\ IES San Juan Bautista (Madrid) \\ Antonio Sánchez Sánchez-Serrano \\ Exprofesor de la Universidad Complutense
}

RESUMEN

Mediante criterios cuantitativos, se estudian detenidamente los usos de las grafías $h, i, y, x, g, j$ en función de su posición en las palabras y teniendo en cuenta las coincidencias y divergencias en las cuatro ediciones consignadas en el título. Del estudio se deducen conclusiones sobre el estado de la ortografía castellana de la época, basadas en los miles de datos extraídos de dichas ediciones, de fácil acceso para los lectores del presente estudio.

Palabras Clave: Pedro Hagenbach, Fadrique de Basilea, Jorge Coci, Juan Joffre, ortografía, tipografía, impresores.

Once more about the orthotypography of the Toledo, 1500 and Burgos, 1499 Comedias and the Zaragoza, 1507 and Valencia, 1514 Tragicomedias. Graphic uses of h, i, y, g, j, x

\section{ABSTRACT}

This paper studies quantitatively the uses of letters $h, I, y, x, g, j$, depending on their position in the word and taking into account the coincidences and divergences in the four editions mentioned in the title. The thousands of data extracted, now made available to the reader, will allow drawing several conclusions relating the state of Castilian spelling at that time.

KeYwords: Pedro Hagenbach, Fadrique de Basilea, Jorge Coci, Juan Joffre, orthography, typography, printing press. 


\section{Para José Luis Canet Vallés}

\section{Preámbulo}

Este artículo es parte integrante de un amplio estudio sobre la materialidad ortotipográfica de las cuatro ediciones citadas en el título que ambos autores estamos realizando en común ${ }^{1}$. Hace ahora cuatro años que dimos a conocer tres fases de este estudio. Los dos primeros artículos están publicados en Celestinesca 38 (2014). En uno abordamos la cuantificación y catalogación de las abreviaturas ${ }^{2}$, lo que nos condujo a reforzar la tesis de la precedencia de la edición de Toledo sobre la de Burgos. En el otro, la evaluación de las erratas ${ }^{3}$ nos llevó a rechazar la atribución de Proaza como corrector de pruebas de imprenta y a defender su función como corrector del manuscrito preparado con anterioridad a su impresión en letras de molde; Proaza habría sido, pues, el censor del manuscrito y sus coplas constituyen la aprobación o censura aprobatoria para poder ser estampado en el taller de imprenta y vendido posteriormente, ejercicio ya en práctica antes de la promulgación de la Pragmática de 1502.

También en 2014, en el Primer Congreso Internacional sobre el Libro Medieval y Moderno celebrado en la Universidad de Zaragoza, presentamos una comunicación titulada "Constricciones y libertades ortográficas de los impresores en cuatro ediciones tempranas de Celestina: Toledo 1500, Burgos 1499-1502 (?), Zaragoza 1507 y Valencia 1514»4, publicada

1.- Como ya explicamos en nuestro primer trabajo (Sánchez Sánchez-Serrano 2014: 125), una de las razones de haber elegido estas cuatro ediciones es de carácter práctico, dado que las cuatro son especialmente accesibles al lector interesado por existir los facsímiles que se indican en la Bibliografía. Registramos la Comedia y la Tragicomedia como anónimas, tal y como eran consideradas en la época (Joseph T. Snow 1999-2000, 2001 y 2005-2006; Remedios Prieto de la Iglesia y Antonio Sánchez Sánchez-Serrano 2016).

2.- Antonio Sánchez Sánchez-Serrano, "Las abreviaturas en cuatro ediciones tempranas de la Celestina: Toledo 1500, Burgos 1499-1502 (?), Zaragoza 1507 y Valencia 1514. Catalogación, cuantificación y consecuencias editoriales».

3.- Remedios Prieto de la Iglesia, «Erratas y corrector de la impresión: Alonso de Proaza y Celestina».

4.- Las grafías de ediciones de la Celestina han sido objeto de la atención de varios estudiosos. Berndt Kelley (1977: 11-14) se detiene en particularidades de la edición de Zaragoza 1507, que Patrizia Botta y Víctor Infantes (1999: 197-198) confirman. Marciales (1985: I, 305-309) muestra cómo la imprenta del periodo rojano se aparta generalmente de las recomendaciones de Nebrija. César García de Lucas (2008) anota algunos aspectos. José Luis Canet (2011: 106109; 2014) muestra las principales peculiaridades de las tres Comedias conservadas, Toledo, Sevilla y Burgos. María Remedios Moralejo Álvarez (2018) establece las características gráficas de la Tragicomedia impresa por Coci en 1545 y la impresa por Pedro Bermuz en 1554, ambas de Zaragoza. Fidel Sebastián (2003) se centra en los signos de puntuación de las tres Comedias y de las Tragicomedias anteriores a 1520. Ivy A. Corfis y John O'Neill (1997) realizan mediante 
en el primer volumen (2015) de la Revista Internacional sobre el Libro Antiguo Titivillus. En aquel entonces catalogamos y cuantificamos en detalle, aunque sin pretender absoluta exactitud matemática dada la multiplicidad de testimonios tenidos en cuenta, las grafías $C, Z, C(+e, i), S C$ $(+e, i)$ de los fonemas sibilantes dentales y el uso de las grafías $B, \mathrm{~V}$ y $U$ atendiendo al lugar que ocuparan en la palabra. Pudimos deducir la existencia de ciertas normas implícitas en el empleo de estas grafías que encasillamos en tres grupos: de carácter ortográfico, de carácter tipográfico y de carácter estético. También observamos cómo los talleres de Pedro Hagenbach, Fadrique de Basilea, Jorge Coci y Juan Joffre obviaban o se alejaban de los criterios ortográficos de Nebrija formulados en su Gramática de la lengua castellana $(1492)^{5}$ en tanto que se acercaban a los que López de Velasco recogería muchos años después en su magnífica y fiable Orthographia y Pronunciación Castellana (1582).

Ahora, en el presente artículo, centraremos nuestra atención en la presencia o ausencia de la $H$ y en los usos de las grafías I, Y, G, J, X en las ediciones de referencia siguiendo sustancialmente los parámetros y la metodología que empleamos en el estudio anterior, del que este es continuación ${ }^{7}$. Nuestro objetivo no estaba ni está encaminado a resolver ningún problema ecdótico o de transmisión impresa (aunque nos congratularíamos de que pudiera servir de humilde instrumento para ello), sino, siguiendo la senda de José Luis Canet, a poner de relieve la contribución de los maestros y oficiales de imprenta a la configuración de normas tipográficas y ortográficas. Nos hacemos eco de sus palabras:

sistemas informáticos el cómputo de las palabras y las concordancias de veinte ediciones de la Celestina transcritas semipaleográficamente en su monumental obra en CD-ROM, aunque sus cuantificaciones no coinciden siempre con las nuestras dado que su metodología y objetivos son otros y sus datos tienen en cuenta tanto los paratextos como el texto literario propiamente dicho, mientras que nosotros solamente contemplamos este (Vid. nota 7).

5.- Nebrija, Antonio de, Gramática de la Lengua Castellana, estudio y edición de Antonio Quilis, pp. 63-65, 111-144.

6.- Las posiciones ortográficas de López de Velasco y la repercusión de su obra en los criterios gramaticales posteriores han sido estudiados por Pozuelo Yvancos (1981), para quien «se trata del más extenso tratado sobre la cuestión de nuestra historia de la lingüística clásica» caracterizada por «su rigor metodológico».

7.- Advertencias: a) Los números entre paréntesis indican la frecuencia de las palabras en el conjunto de las cuatro ediciones; b) las cuantificaciones se realizan sobre el texto puramente literario, prescindiendo de los paratextos iniciales y finales dada la falta de uniformidad entre las cuatro ediciones; c) para el cómputo de palabras solo se tiene en cuenta la cuestión que en ese momento se está examinando y tomando en cuenta la categoría gramatical de las palabras homónimas; d) en las cuantificaciones no se incluyen las palabras con erratas si estas afectan de forma inequívoca al caso en estudio; e) en las formas especiales, indicamos su localización en el impreso poniendo su signatura tipográfica entre corchetes: letra del cuaderno y número de la plana, recto (r) y vuelto (v) seguido del número de la línea; f) en las transcripciones de palabras desarrollamos las abreviaturas si estas no afectan a la cuestión que se está tratando en ese momento, en cuyo caso no se contabiliza la palabra. 
La bibliografía textual abre nuevas vías para una mejor comprensión de la evolución de la lengua y ortografía de la lengua castellana, teniendo en este proceso más importancia los correctores y componedores de imprenta (algunas veces esta función la realizarán los impresores) que los propios autores, al menos desde el nacimiento de la imprenta hasta mediados del XVI. (Canet 2005: 380)

\section{Usos de la grafía $H$}

\section{H- en posición inicial de palabra}

En el conjunto de las cuatro ediciones, 874 palabras coinciden en llevar $h$ - en posición inicial invariablemente, sin excepción alguna. Atendiendo a su número, ya que esto constituye un índice de fiabilidad, los ejemplos más importantes son: hombre/s (377), hermano y derivados (160), humano y derivados (45) y hembra/s (26). Cabe incluir también en este grupo el verbo honrrar y el sustantivo honrra, con 192 testimonios, no contabilizados en la cifra primera por el hecho de que sus derivados deshonrrado y deshonrra omiten la $-h$ - interior en cuatro de las doce veces que aparecen: dos en Burgos $\left[1_{j}{ }_{j}^{r: 3}, 1_{j}{ }^{r: 25}\right]$ y dos en Valencia $\left[g_{v}{ }_{v}^{v: 33}, g_{v j}{ }^{r: 1}\right]^{8}$.

Más digno de reflexión resulta el caso de huertola con 62 testimonios, siempre con $h$-inicial. Sin embargo sorprende que sus derivados ortolano, ortelano, ortelana y ortaliza se escriban $\sin h$ - en las 10 veces que hemos registrado entre las cuatro ediciones ${ }^{9}$. Por supuesto que la etimología (del latín hortus) justifica el uso de la h-, pero por la misma razón haría necesario el comienzo por $h$ - de los derivados mencionados.

Por otra parte, el ossum latino no justifica la utilización de la $h$-inicial en las 20 ocasiones en que aparece la palabra huesso/s. Según aclara López de Velasco ${ }^{10}$, el uso de la $h$ - en los vocablos que estamos analizando se debe a su comienzo con el diptongo hue-, como se percibe también y más claramente en la contraposición entre olorles, oler, olías, olorosas (30), y huele (8), si bien por unas razones de carácter tipográfico completamente ajenas a las costumbres actuales: si huerto, hueso, huele y otras palabras con el mismo comienzo ${ }^{11}$ se escribieran $\sin h$-, la letra inicial sería $U$ - que, de

\section{8.- Véase nota anterior.}

9.- Ortolano en Toledo [f iij r:18-19], Burgos [f viij v:19] y Valencia [d vij v:32]; ortelano en Zaragoza [g iiij r:17-18] y Valencia [f viij r:13]; ortelana en Zaragoza [e ij v:34, i iij v:15] y Valencia [h vij r:35]; ortaliza en Zaragoza [g iiij r:20] y Valencia [f viij r:15].

10.- Juan López de Velasco, Orthographia y pronunciación castellana, Burgos, 1582, pp. 138-139.

11.- Como por ejemplo huelgals, huellas, huesped o huestantigua. 
acuerdo con las costumbres tipográficas de entonces ${ }^{12}$, se convertiría en $V$-, dando lugar a verto, vessos y vele, propiciando dudas sobre la pronunciación de dicha $v$-como consonante o como vocal y por tanto sobre la identificación de la palabra sobre todo en la lectura declamatoria en voz alta a la que alude Proaza en sus coplas finales cuando indica el «modo que se ha de tener leyendo» la Celestina.

\section{Presencia o ausencia de $\mathrm{H}$ - en el verbo (h)auer y en otras palabras}

Dentro de este apartado alcanza singular relieve el verbo hauer o auer. La lectura, con cierta atención a las grafías, de las cuatro ediciones objeto de nuestro estudio, sugiere la idea de un uso arbitrario de la $h$ - como letra inicial de la flexión de dicho verbo. Sin embargo, la cuantificación de sus formas permite detectar algunas normas o, quizá, solo costumbres que, como hemos dicho en otros lugares ${ }^{13}$, parecen tener más relación con los aspectos gráficos y estéticos que con los ortográficos o etimológicos.

La primera evidencia de ello es que, en su mayoría, la utilización de la $h$ - inicial está relacionada con la brevedad de la palabra y el presente de indicativo: 1477 con $h$ - frente a 114 en que se omite. La inmensa mayoría de estas excepciones se produce en la $2^{\mathrm{a}}$ persona del singular as (109), correspondiendo 78 de ellas a la edición de Zaragoza, en la que superan a la forma con $h$ - (52). Como nota adicional añadimos que hemos aparece en 12 ocasiones en total, sin que ni una sola vez se haya escrito $\sin h$ - (Vid. Tabla $\left.{ }^{\circ} 1\right)^{14}$. Resumiendo: el predominio de la $h$ - supone el $92,83 \%$ frente al $7,16 \%$ sin ella.

\section{Tabla $\mathrm{n}^{\circ} 1$}

12.- Como es sabido, V y U son dos figuras de una misma letra con el doble valor de consonante y vocal, utilizándose una u otra figura dependiendo del lugar que ocupe en las palabras: $\mathrm{U}$ en el interior y $\mathrm{V}$ al principio. Véase nuestro artículo "Constricciones y libertades ortográficas de los impresores...», pp. 244-248. López de Velasco (1582: 153) es claro al respecto: se pone h- "para impedir que la i y la u vocales quando se le siguen otras vocales en el principio $[\ldots]$ no se conviertan en consonantes».

13.- Remedios Prieto de la Iglesia, «Erratas y corrector de la impresión: Alonso de Proaza y Celestina», Celestinesca 38 (2014), pp. 113-124. Antonio Sánchez Sánchez-Serrano, «Las abreviaturas en cuatro ediciones tempranas de la Celestina: Toledo 1500, Burgos 1499-1502 (?), Zaragoza 1507 y Valencia 1514», Celestinesca 38 (2014), pp. 125-154. Remedios Prieto de la Iglesia, "Constricciones y libertades ortográficas de los impresores en cuatro ediciones tempanas de Celestina: Toledo 1500, Burgos 1499-1502 (?), Zaragoza 1507 y Valencia 1514», Titivillus 1 (2015), pp. 243-255.

14.- A veces se verá falta de coincidencia en las sumas de las cuantificaciones, ya en la versión Comedia de 16 autos (Toledo y Burgos), ya en la versión Tragicomedia de 21 (Zaragoza y Valencia). Ello se debe a la existencia de otras lecturas excepcionales (variantes o erratas) o a la inexistencia de los argumentos de los autos en la edición de Zaragoza. Se señalará la localización de estas excepciones indicando la signatura tipográfica del impreso: letra del cuaderno y número de la plana, recto (r) y vuelto (v) seguido del número de la línea en que se halla. 


\begin{tabular}{|l|c|c|c|c|c|}
\hline & Toledo & Burgos & Zaragoza & Valencia & Total \\
\hline he & 95 & $94^{14 . a}$ & 104 & 104 & 397 \\
\hline e & 0 & 0 & 0 & 0 & 0 \\
\hline has & $106^{14 . b}$ & 95 & 52 & $123^{14 . c}$ & 376 \\
\hline as & 7 & 19 & 78 & $51^{14 . d}$ & 109 \\
\hline ha & 124 & 123 & 153 & 157 & 557 \\
\hline a & 0 & 1 & 3 & 0 & 4 \\
\hline hemos & 2 & 2 & 4 & 4 & 12 \\
\hline emos & 0 & 0 & 0 & 0 & 0 \\
\hline han & 30 & 30 & 37 & 38 & 135 \\
\hline an & 0 & 0 & 1 & 0 & 1 \\
\hline
\end{tabular}

Por contraste, en todos los demás tiempos predomina la carencia de $h$ - inicial: $1020 \sin h$-frente a 260 con ella, es decir, el 79,69\% y el $20,31 \%$ respectivamente. Dignos de resaltar son los siguientes condicionamientos:

A) Formas que comienzan por el grupo de letras $a+y$ (Vid. Tabla $\left.n^{\circ} 2\right)$.

- Impersonal del presente de indicativo: $349 \sin h$-y 11 con ella.

- Presente de subjuntivo: $150 \sin h$-y 20 con ella.

\section{Tabla $\mathrm{n}^{\circ} 2$}

\begin{tabular}{|l|c|c|c|c|c|}
\hline & Toledo & Burgos & Zaragoza & Valencia & Total \\
\hline ay & 73 & 78 & 96 & 102 & 349 \\
\hline hay & 5 & 0 & 6 & 0 & 11 \\
\hline aya $\left(1^{a}\right.$ p.) & 6 & 7 & 5 & 6 & 24 \\
\hline haya $\left(1^{a}\right.$ p.) & 1 & 0 & 2 & 1 & 4 \\
\hline ayas & 6 & $6^{14 . e}$ & 6 & 7 & 25 \\
\hline hayas & 1 & 0 & 1 & 0 & 2 \\
\hline aya $\left(3^{a}\right.$ p.) & 18 & $17^{14 . f}$ & 14 & $23^{14 . g}$ & 72 \\
\hline haya $\left(3^{a}\right.$ p.) & 0 & 0 & 9 & 1 & 10 \\
\hline
\end{tabular}

14.a.- Burgos, en lugar de "el vientre no se le he visto», escribe «el vientre no se le visto» [h ij r:27].

14.b.- En Toledo, en lugar de «has pensado», «vas pensando» [a viij v:27].

14.c.- En Valencia, en lugar de "que la has tocado dizes», "que la ha tocado dizes» [d iij v:2].

14.d.-En Valencia, en lugar de "qué (h)as mi elicia», "qué es mi elicia» [a viij r: 29-30].

14.e.- En Burgos en lugar de ayas, oyas [f vij v:6].

14.f.- En Burgos en lugar de aya, ay [k vj v:2].

14.g.- En Valencia una más pues se encuentra en el Argumento del Auto XV [g vij ${ }^{\text {t:39}}$ ], inexistente en la edición de Zaragoza. 


\begin{tabular}{|l|c|c|c|c|c|}
\hline ayamos & $1^{14 . \mathrm{h}}$ & 2 & 2 & 2 & 7 \\
\hline hayamos & 1 & 0 & 0 & 0 & 1 \\
\hline ayan & 5 & 4 & 5 & $8^{14 . \mathrm{i}}$ & 22 \\
\hline hayan & 0 & 1 & 2 & 0 & 3 \\
\hline
\end{tabular}

El hecho de que el impersonal del presente se escriba tan mayoritariamente $\sin h$ - se contrapone a la circunstancia advertida de que el uso de esta letra parezca vinculado a la brevedad de la palabra y a dicho tiempo. Pero en este caso se vislumbra otra tendencia: la de homogeneizar ortografías y sonidos para evitar errores y confusionismos tipográficos. Quizá se deba a esto que el adverbio de lugar ay (actual ahi) y la interjección ay que en la actualidad escribimos entre signos de admiración coincidan en su escritura entre sí y con la forma impersonal de la que ahora nos ocupamos. El adverbio aparece en 49 ocasiones, si bien dos en Toledo [ $\mathrm{a}_{\mathrm{iiij}}^{\mathrm{r}: 13}$, e ${ }_{\text {vij }}^{\text {r: } 1}$ ] y una en Burgos $\left[a_{j}{ }_{j}\right.$ : 16$]$ se sustituye por la forma arcaica $y$, mientras que encontramos la interjección 93 veces, aunque Toledo $\left[\mathrm{c}_{j}\right.$ v: 25$]$ y Burgos $\left[\mathrm{C}_{\mathrm{ij}}{ }^{\mathrm{r}: 28}\right]$ escriben una vez hay exactamente en el mismo lugar del texto.

Por cuestión de analogía nos ocupamos aquí del adverbio de tiempo hoy u oy, cuyas frecuencias registramos en el siguiente cuadro:

\begin{tabular}{|l|c|c|c|c|c|}
\hline & Toledo & Burgos & Zaragoza & Valencia & Total \\
\hline oy & 23 & 25 & 4 & 25 & 77 \\
\hline hoy & 1 & 0 & 22 & 1 & 24 \\
\hline Totales & 24 & 25 & 26 & 26 & 101 \\
\hline
\end{tabular}

del que podemos deducir la discrepancia en cuanto a la ortografía entre la edición de Zaragoza, que se atiene, aunque con cuatro excepciones, a la etimología del hodie latino, y las tres restantes que, prescindiendo de la etimología, identifican su escritura al pretérito indefinido oy del verbo oyr.

B) Tiempos que incluyen - $U$ - consonante en su interior: infinitivo, gerundio, participio, presente de indicativo, pretérito imperfecto de indicativo, futuro de indicativo y condicional (Vid. Tabla $n^{\circ} 3$ ). De las 187 veces que se utiliza la $h$ - (frente a las 403 que carecen de ella), 101 se producen en la edición de Burgos que, en cambio, solo omite dicha letra en 27. 
Tabla $n^{\circ} 3$

\begin{tabular}{|c|c|c|c|c|c|}
\hline & Toledo & Burgos & Zaragoza & Valencia & Total \\
\hline auer & $21^{14-j}$ & $6^{14 . k}$ & $28^{14.1}$ & $36^{14 . m}$ & 91 \\
\hline hauer & 11 & 25 & 8 & 4 & 48 \\
\hline auiendo & 1 & 0 & 2 & 3 & 6 \\
\hline hauiendo & 1 & 2 & 1 & 0 & 4 \\
\hline auido & 5 & 1 & 6 & 6 & 18 \\
\hline hauido & 1 & 5 & 1 & 1 & 8 \\
\hline auemos & 1 & 1 & 3 & 6 & 11 \\
\hline hauemos & 2 & 2 & 3 & 0 & 7 \\
\hline aues o aueys & 10 & 4 & 11 & 13 & 38 \\
\hline haues o haueys & 2 & 8 & 2 & 0 & 12 \\
\hline auia $\left(1^{\mathrm{a}} \mathrm{p}.\right)$ & 6 & 3 & 10 & 9 & 28 \\
\hline hauia $\left(1^{\mathrm{a}} \mathrm{p}.\right)$ & 5 & 8 & 2 & 3 & 18 \\
\hline auias & $12^{14 \cdot \mathrm{n}}$ & 6 & 13 & 14 & 45 \\
\hline havias & 1 & 7 & 3 & 2 & 13 \\
\hline auia $\left(3^{\mathrm{a}} \mathrm{p}.\right)$ & $13^{14.0}$ & 3 & 25 & 2914.p & 70 \\
\hline hauia ( $\left.3^{\mathrm{a}} \mathrm{p}.\right)$ & 8 & 18 & 7 & 7 & 40 \\
\hline auiemos & 2 & 0 & 2 & $2^{14 . q}$ & 6 \\
\hline hauiamos & 0 & 2 & 0 & 0 & 2 \\
\hline auiades & 0 & 0 & 1 & 1 & 2 \\
\hline hauiades & 0 & 0 & 0 & 0 & 0 \\
\hline auian & 4 & 1 & $4^{14 . r}$ & $8^{14 . s}$ & 17 \\
\hline hauian & 1 & 4 & 1 & 0 & 6 \\
\hline aure & 2 & 0 & 3 & 4 & 9 \\
\hline haure & 0 & 2 & 1 & 0 & 3 \\
\hline auras & 6 & 1 & 6 & 7 & 20 \\
\hline
\end{tabular}

14.j.- Toledo presenta la lectura auer [f vij v:18] donde las demás ediciones escriben ver. 14.k.- En Toledo dos veces aver [e viij r:13, f viij r:17].

14.1.- En Zaragoza auie [a v v:8] en lugar de auer.

14.m.- En Valencia dos más que en Zaragoza pues se encuentran en los Argumentos de los Autos VII $\left[\mathrm{d}_{\mathrm{v}}{ }_{\mathrm{v}}^{\mathrm{r}: 5}\right]$ y XIV $\left[\mathrm{g}_{\mathrm{iiij}}^{\mathrm{r}: 40}\right]$, inexistentes en Zaragoza.

14.n.- En Toledo una vez avias [f vj r:2].

14.o.- En Toledo una vez avia [d v r:4].

14.p.- En Valencia cuatro más que en Zaragoza pues se encuentran en los Argumentos de los Autos XII [hauia f v r: 36], XIV [g iiij r:37], XIX [h vj v:5] y XX [i j r:3], inexistentes en Zaragoza.

14.q.- En Valencia una vez auiamos [g j r:35].

14.r.- En Zaragoza auia [i j v:6] y veia [h iiij v:31] en lugar de auian.

14.s.- En Valencia una más en el Argumento del Auto XV [g vij ${ }_{\text {rija }}$ ]. 


\begin{tabular}{|l|c|c|c|c|c|}
\hline hauras & 0 & 5 & 1 & 0 & 6 \\
\hline aura & $5^{14 . t}$ & 0 & 9 & 9 & 23 \\
\hline haura & 3 & 8 & 1 & 1 & 13 \\
\hline auremos & 2 & 1 & 2 & 2 & 7 \\
\hline hauremos & 0 & 1 & 0 & 0 & 1 \\
\hline aurias & 1 & 0 & 2 & 2 & 5 \\
\hline haurias & 1 & 2 & 0 & 0 & 3 \\
\hline auria & 2 & 0 & 2 & 3 & 7 \\
\hline hauria & 0 & 2 & 1 & 0 & 3 \\
\hline
\end{tabular}

Las cifras globales y los porcentajes son en este caso los siguientes:

\begin{tabular}{|l|c|c|c|c|c|}
\hline & Toledo & Burgos & Zaragoza & Valencia & Total \\
\hline Sin $h$-inicial & 93 & 27 & 129 & 154 & 403 \\
& $(72,09 \%)$ & $(21,09 \%)$ & $(80,12 \%)$ & $(89,53 \%)$ & $(68,38 \%)$ \\
\hline Con $h$ - inicial & 36 & 101 & 32 & 18 & 187 \\
& $(27,90 \%)$ & $(78,90 \%)$ & $(19,87 \%)$ & $(10,46 \%)$ & $(31,69 \%)$ \\
\hline
\end{tabular}

En ello se observa que en las Tragicomedias las formas de este grupo que carecen de $h$-inicial superan el $80 \%$, la Comedia de Toledo rebaja la diferencia, mientras que la de Burgos invierte los términos: 78,90\% para las formas con $h$-.

Finalmente, como caso particular del anterior debido al cambio de la vocal inicial, consideraremos el pretérito fuerte y tiempos de subjuntivo que de él derivan, que además de llevar -u-consonante interior no comienzan por $a$ - sino por o- (Vid. tabla $\left.{ }^{\circ} 4\right)$. También en este caso, la inmensa mayoría de las formas con $h$ - inicial se encuentran en la edición de Burgos: 31 de 39 en total ${ }^{15}$.

Tabla $\mathrm{n}^{\circ} 4$

\begin{tabular}{|l|c|c|c|c|c|}
\hline & Toledo & Burgos & Zaragoza & Valencia & Total \\
\hline oue & 6 & 2 & 6 & $3^{15 . b i s}$ & 17 \\
\hline houe & 0 & 4 & 0 & 0 & 4 \\
\hline ouiste & 0 & 0 & 1 & 1 & 2 \\
\hline houiste & 1 & 1 & 0 & 0 & 2 \\
\hline ouo & 8 & 0 & 8 & 10 & 26 \\
\hline houo & 1 & 9 & 3 & 1 & 14 \\
\hline
\end{tabular}




\begin{tabular}{|c|c|c|c|c|c|}
\hline ouieron & 0 & 0 & 2 & 2 & 4 \\
\hline houieron & 0 & 0 & 0 & 0 & 0 \\
\hline ouiera (1 $\left.{ }^{\mathrm{a}} \mathrm{p}.\right)$ & 2 & 0 & 2 & 2 & 6 \\
\hline houiera $\left(1^{a} p.\right)$ & 0 & 2 & 0 & 0 & 2 \\
\hline ouieras & 3 & 0 & 3 & 3 & 9 \\
\hline houieras & 0 & 3 & 0 & 0 & 3 \\
\hline ouiera ( $\left.3^{\mathrm{a}} \mathrm{p}.\right)$ & 2 & 0 & 2 & 2 & 6 \\
\hline houiera ( $\left.3^{\mathrm{a}} \mathrm{p}.\right)$ & 0 & 2 & 0 & 0 & 2 \\
\hline ouiesse $\left(1^{\mathrm{a}} \mathrm{p}.\right)$ & 1 & 1 & 2 & 2 & 6 \\
\hline houies $(s)$ e $\left(1^{\mathrm{a}} \mathrm{p}\right.$.) & 1 & 1 & 0 & 0 & 2 \\
\hline ouiesses & 1 & 0 & 1 & 0 & 2 \\
\hline houiesses & 0 & 1 & 0 & 1 & 2 \\
\hline ouies(s)e ( $3^{\mathrm{a}}$ p.) & 5 & 0 & 7 & 7 & 19 \\
\hline houies(s)e ( $3^{\mathrm{a}}$ p.) & 0 & 5 & 0 & 0 & 5 \\
\hline ouies(s)emos & 0 & 0 & 1 & 1 & 2 \\
\hline houies(s)emos & 0 & 0 & 0 & 0 & 0 \\
\hline ouiessen & 1 & 0 & 1 & 1 & 3 \\
\hline houiessen & 0 & 1 & 0 & 0 & 1 \\
\hline ouieres & 0 & 0 & 1 & 1 & 2 \\
\hline houieres & 0 & 0 & 0 & 0 & 0 \\
\hline oniere ( $\left.3^{\mathrm{a}} \mathrm{p}.\right)$ & 3 & 2 & 3 & 3 & 11 \\
\hline houiere ( $3^{\mathrm{a}}$ p.) & 0 & 1 & 0 & 0 & 1 \\
\hline ouieremos & 1 & 0 & 1 & 1 & 3 \\
\hline houieremos & 0 & 1 & 0 & 0 & 1 \\
\hline
\end{tabular}

En este otro grupo, las cifras y porcentajes acentúan las diferencias que se daban en el anterior:

\begin{tabular}{|l|c|c|c|c|c|}
\hline & Toledo & Burgos & Zaragoza & Valencia & Total \\
\hline Sin $h-$ & 33 & 5 & 41 & 39 & 118 \\
inicial & $(91,66 \%)$ & $(13,88 \%)$ & $(93,18 \%)$ & $(95,12 \%)$ & $(75,15 \%)$ \\
\hline Con $h-$ & 3 & 31 & 3 & 2 & 39 \\
inicial & $(8,33 \%)$ & $(86,11 \%)$ & $(6,81 \%)$ & $(4,87 \%)$ & $(24,84 \%)$ \\
\hline
\end{tabular}

Asimismo, esta alternancia entre utilización o supresión de la $h$-inicial se da en otras palabras, si bien vamos a hacer referencia solo a tres casos: los dos primeros en función de la frecuencia y el último por su interés.

1. El sustantivo hora (166), cuya distribución es: 


\begin{tabular}{|l|c|c|c|c|c|}
\hline & Toledo & Burgos & Zaragoza & Valencia & Total \\
\hline Con $h$-inicial & 30 & 35 & 46 & 45 & 156 \\
\hline Sin $h$ - inicial & 7 & 2 & 0 & 1 & 10 \\
\hline Totales & 37 & 37 & 46 & 46 & 166 \\
\hline
\end{tabular}

2. Honesto y derivados (68) con el siguiente reparto:

\begin{tabular}{|l|c|c|c|c|c|}
\hline & Toledo & Burgos & Zaragoza & Valencia & Total \\
\hline honest(id)ad & 9 & 6 & 10 & 10 & 35 \\
\hline onest(id)ad & 0 & 3 & 0 & 0 & 3 \\
\hline honesto/a/as & 2 & 0 & 8 & 8 & 18 \\
\hline onesto/a & 0 & 2 & 0 & 0 & 2 \\
\hline deshonesta/os/as & 2 & 1 & 3 & 3 & 9 \\
\hline desonesta & 0 & 1 & 0 & 0 & 1 \\
\hline
\end{tabular}

en donde se ve que la supresión de la $h$ es cuestión exclusiva de la edición de Burgos, si bien el número de casos resulta reducido para extraer conclusiones aceptables.

3. Por último resaltamos dos secuencias: historiales e historias en Toledo $\left[\mathrm{a}_{\mathrm{vi}}^{\mathrm{v}: 32}, \mathrm{c}_{\mathrm{ij}}^{\mathrm{v}: \mathrm{:}}\right]$, e ystoriales e ystorias en Burgos $\left[\mathrm{a}_{\mathrm{v}}^{\mathrm{v}: 12}, \mathrm{c}_{\mathrm{iij}}^{\mathrm{r}: 20}\right]$ y Zaragoza [a ri: $15, b_{\text {vij }}$ : 28$]$, en las que la supresión de la $h$ - precisa una sustitución de la - $i$ - latina por $y$ - griega puesto que las costumbres tipográficas de la época, según se observa en las cuatro ediciones examinadas, no aceptaban palabras iniciadas por $i$ - latina con valor vocálico si no lo eran por los prefijos de negación o locativo in-, im-, i-, bien de formación romance o bien incluidos primitivamente en una raíz latina.

\section{Intercambio entre $\mathrm{H}-y$ F- en posición inicial de palabra}

La mayor parte de las alternancias entre $h$ - y $f$-iniciales podrían ser consideradas excepciones al apartado anterior, dada la desproporción, siempre favorable a la $h$ - salvo en un solo caso que se verá, en el empleo de ambas letras iniciales según se evidencia en los siguientes ejemplos que arrojan un porcentaje de $93,79 \%$ con $h$ - sobre $6,21 \%$ con $f$-:

- hijo/alos/as (437) frente a fijo/alos/as (35). Las variantes apocopadas hi (3), hy (3) y fi (4) ${ }^{16}$ están equilibradas pero su número es tan escaso que no permite sacar conclusiones mínimamente fiables.

- Las diversas formas de la conjugación del verbo holgar se escriben con $h$ - inicial 128 veces y solo 2 con $f$-: folgar en Burgos [c $\left.{ }_{v j}^{v: 1-2}\right]$ y 
folgado en Valencia [ $\mathrm{c}_{\text {iiij }}{ }^{\mathrm{r}: 33-34}$. No obstante, el sustantivo folgura, testimoniado una sola vez en el texto, está escrito con $f$ - en Toledo $\left[f_{v j}{ }^{r .5}\right]$, Burgos $\left[g_{i i j}{ }^{v: 2}\right]$ y Valencia $\left[e_{j}{ }_{j: 16}\right]$ y únicamente con $h$ - en Zaragoza [e ${ }_{\mathrm{v}}^{\mathrm{r}: 1}$ ].

- Algo similar ocurre en los siguientes ejemplos dignos de atención por sus frecuencias:

- huyr, 120 con $h$ - y 3 con $f$ - en Zaragoza [b ${ }_{j}^{\text {v:16] }}$ y Valencia [a viij $\mathrm{f}_{\mathrm{vj}}^{\mathrm{r}: 42}$;

- huyda (11) frente a fuyda (1) en Valencia $\left[\mathrm{f}_{\mathrm{vij}}^{\mathrm{v}: 32}\right.$;

- hasta (149), fasta (14) en Toledo [b ${ }_{\mathrm{ij}}^{\mathrm{r}: 2}, \mathrm{c}$ vij ${ }_{\mathrm{v}}^{\mathrm{v} 9}, \mathrm{~d}_{\mathrm{ij}}^{\mathrm{v}: 32}, \mathrm{~g}_{\mathrm{j}}^{\mathrm{v}: 21}$, Burgos $\left[a_{j}^{r: 28}, c_{i i i j}^{v: 21}, d_{j}^{r: 14}, d_{v}^{r: 24}, e_{v i j}^{r: 27-28}, g_{v i j}^{v: 25}, m_{j}^{r: 27}\right]$ y Valencia $\left[b_{\text {iij }}\right.$ v:2,$b_{\text {iiij }}$ ::8,$e_{j}$ r:16];

- hermosola y hermosura (85), fermosa y fermosura (9) en Toledo [a ${ }_{\text {iiii }}^{\mathrm{r}: 32}$,

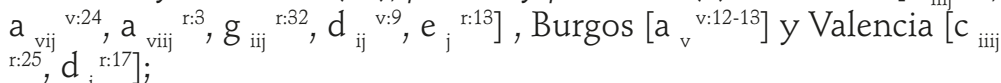

- verbo herir y derivados (43), ferida (1) en Valencia [ $\mathrm{d}_{\mathrm{iiij}}^{\mathrm{r}: 17}$;

- hoja (23), foja (1) en Toledo [b ${ }_{\text {iij }}^{\text {v:24]; }}$

- hilado, hilos e hilar (66), filado y filar (6) en Toledo $\left[\mathrm{e}_{\mathrm{ij}}^{\mathrm{v}: 21}, \mathrm{e}_{\mathrm{iij}}^{\mathrm{r}: 1}, \mathrm{~b}_{\mathrm{iij}}^{\mathrm{r}: 16}\right]$, Burgos $\left[e_{\mathrm{ij}}^{\mathrm{v}: 12}, \mathrm{~b}_{\mathrm{ij}}^{\mathrm{v}: 6}\right]$ y Valencia $\left[\mathrm{c}_{\mathrm{iij}}^{\mathrm{r}: 22}\right]$;

- haldas y haldear (33), faldas (1) en Valencia [ $\left.\mathrm{f}_{\mathrm{vij}} \mathrm{v}: 32\right]$;

- hazienda/s (38), fazienda (2) en Toledo [ $\mathrm{e}_{\text {viij }}$ r:14] y Burgos $\left[\mathrm{f}_{\mathrm{v}} \mathrm{r}: 22\right]$.

Hay que resaltar sin embargo el caso de fuego (41) frente a huego (15), con la particularidad añadida de que 12 de estas 15 variantes están en la edición de Zaragoza ${ }^{17}$.

Asimismo se alterna el uso de la $h$ - y $f$ - iniciales en la conjugación del verbo hazer o fazer, si bien también aquí el número de las palabras que empiezan por $h$ - supera muy ampliamente al de las que lo hacen por $f$-. Según el recuento realizado, los datos comparativos entre hazer y fazer son:

\begin{tabular}{|l|c|c|c|c|c|}
\hline & Toledo & Burgos & Zaragoza & Valencia & Total \\
\hline $\begin{array}{l}\text { Formas } \\
\text { con } h-\end{array}$ & $\begin{array}{c}273 \\
(82,48 \%)\end{array}$ & $\begin{array}{c}239 \\
(72,21 \%)\end{array}$ & $\begin{array}{c}432 \\
(98,86 \%)\end{array}$ & $\begin{array}{c}407 \\
(91,05 \%)\end{array}$ & $\begin{array}{c}1351 \\
(87,39 \%)\end{array}$ \\
\hline $\begin{array}{l}\text { Formas } \\
\text { con } f-\end{array}$ & 58 & 92 & 5 & 40 & 195 \\
\hline Totales & $331,52 \%)$ & $(27,79 \%)$ & $(1,14 \%)$ & $(8,94 \%)$ & $(12,61 \%)$ \\
\hline
\end{tabular}


Se constata a través de estos datos que las Tragicomedias utilizan la $h$ - de manera casi absoluta, sobre todo la edición de Zaragoza ${ }^{18}$, mientras que la Comedia de Burgos usa la $f$ - en algo más de la cuarta parte de las ocasiones y la de Toledo también en una proporción considerable ${ }^{19}$.

En el sustantivo hecho y su plural estas diferencias no son tan acusadas:

\begin{tabular}{|c|c|c|c|c|c|}
\hline & Toledo & Burgos & Zaragoza & Valencia & Total \\
\hline $\begin{array}{l}\text { Formas } \\
\text { con } h \text { - }\end{array}$ & $\begin{array}{c}6 \\
(60,00 \%)\end{array}$ & $\begin{array}{c}5 \\
(50,00 \%)\end{array}$ & $\begin{array}{c}11 \\
(100,00 \%)\end{array}$ & $\begin{array}{c}11 \\
(91,67 \%)\end{array}$ & $\begin{array}{c}33 \\
(76,44 \%)\end{array}$ \\
\hline $\begin{array}{l}\text { Formas } \\
\text { con } f \text { - }\end{array}$ & $\begin{array}{c}4 \\
(40,00 \%)\end{array}$ & $\begin{array}{c}5 \\
(50,00 \%)\end{array}$ & $\begin{array}{c}0 \\
(0,00 \%)\end{array}$ & $\begin{array}{c}1 \\
(8,33 \%)\end{array}$ & $\begin{array}{c}10 \\
(23,56 \%)\end{array}$ \\
\hline Totales & 10 & 10 & 11 & 12 & 43 \\
\hline
\end{tabular}

En realidad, son solo las cifras de las Tragicomedias las que propician la desproporción porque las de las Comedias resultan bastante equilibradas.

El último caso interesante, en función de sus numerosos testimonios, es el verbo hablar y los sustantivos de él derivados habla/s y hablilla/s. Solo diremos que sigue la norma general: 596 con $h$ - inicial frente a 78 con $f$-.

Los derivados compuestos mediante anteposición de un prefijo conservan la $h$ en el interior de la palabra con una única excepción en la edición de Zaragoza: desecho [a ${ }_{\text {vij }}^{\text {v:14] }}$; en cambio esta misma edición escribe deshecho $\left[\mathrm{b}_{\mathrm{iij}} \mathrm{v}: 34-35\right]$ así como deshaziendo $\left[\mathrm{i}_{\mathrm{iiij}} \mathrm{r:33}\right]$, deshago $\left[\mathrm{i}_{\mathrm{iij}}^{\mathrm{v}: 19}\right]$ y deshaze $\left[\mathrm{c}_{\mathrm{j}}^{\mathrm{v}: 17}\right]$, además de malhechora $\left[\mathrm{d}_{\mathrm{v}}^{\mathrm{r}: 6}\right]$ y malhechores $\left[\mathrm{g}_{\mathrm{vij}} \mathrm{v}: 28, \mathrm{~h}_{\mathrm{ij}}^{\mathrm{v}: 9}\right]$.

\section{H- inicial, supresión o sustitución por F-}

Esta circunstancia se produce en un reducido número de palabras, siendo absolutamente predominante el uso de la $h$-como se patentiza en las siguientes cifras:

\begin{tabular}{|l|c|c|c|c|}
\hline & con $h$ - & $\begin{array}{c}\text { supresión } \\
\text { de } h-\end{array}$ & $\begin{array}{c}\text { sustitución } \\
\text { por } f \text { - }\end{array}$ & total \\
\hline Verbo hallar & 148 & 5 & 18 & 171 \\
\hline harto/a & 90 & 2 & 8 & 100 \\
\hline hechizera y derivados & 42 & 1 & 1 & 44 \\
\hline hierro & 12 & 1 & 1 & 14 \\
\hline
\end{tabular}

teniendo lugar en esta última palabra la misma mutación de la $i$ - latina inicial que hemos visto en ystoriales e ystorias, que produciría la sustitución de la $i$ - por la $y$ - que da lugar a la grafía yerro. 


\section{$\mathrm{H}-$ en posición interior de palabra}

En páginas anteriores hemos señalado lo que ocurre con algunas palabras formadas mediante la anteposición de un prefijo a otras comenzadas por $h$-como deshonrra o desonrra, deshonrrado o desonrrado y deshonesta o desonesta.

Hay además algunas otras que se escriben con - $h$ - interior sin divergencia alguna aunque su número resulta insuficiente para extraer conclusiones, excepto en el caso de alcahueta que junto con sus derivados suman 34 .

Por el contrario, las formas del verbo reprehender pierden las letras -heen cuatro de las 32 ocasiones en que aparecen.

Finalmente, aunque se trate de proporciones minoritarias, merece la pena sacar a colación lo que ocurre en la flexión de los verbos traer y caer cuando se juntan las vocales a y e. En el primero de ellos esto se produce 139 veces, pero en 8 con $-h$ - intercalada: traher en Toledo [ $\mathrm{c}_{\mathrm{viij}}{ }_{\mathrm{v}: 9}, \mathrm{f}_{\mathrm{ij}}^{\mathrm{r}: 13}$ ] y Burgos [ $b_{i j}^{r: 29}$ ] y trahe en Toledo [ $\mathrm{c}_{\mathrm{iij}}^{\mathrm{r}: 5}, \mathrm{c}_{\mathrm{iiij}}^{\mathrm{v}: 10}, \mathrm{e}_{\mathrm{vj}}^{\mathrm{v}: 30}, \mathrm{~h}_{\mathrm{j}}^{\mathrm{r}: 8}$, ] y $\mathrm{Za}$ ragoza $\left[\mathrm{d}_{\mathrm{ij}}^{\mathrm{r}: 14}\right]$. En el segundo encontramos tres veces cahe en Toledo $\left[b_{\text {ij }}^{\text {r:21 }}, b_{\text {vij: }}^{\text {v:11, }} i_{\text {vj }}^{v: 4}\right]$ mientras que las formas sin - $h$ - interior alcanzan la cifra de 52. Como vemos, esto afecta fundamentalmente a la edición toledana y acaso no se trate simplemente de erratas o de un recurso del cajista para justificar las líneas, sino reflejo de un fenómeno aludido por López de Velasco (1582: 149): "Por escusar hiatos que las vocales hazen juntas, se interpone la $h$ en algunas palabras donde se juntan dos».

\section{Usos de las grafías $I, Y$ con valor vocálico}

\section{Conjunción copulativa}

La Y conjunción copulativa mayúscula es utilizada con carácter exclusivo solo en la edición de Zaragoza (75). Por el contrario, las ediciones de Burgos y Valencia prefieren la E mayúscula (66 y 100 respectivamente). Únicamente la edición de Toledo alterna ambas formas: 81 veces la Y y 12 la E. Las disparidades de los números se deben al uso más bien discrecional de mayúsculas y minúsculas, sin seguir propiamente criterios ortográficos y dependiendo en gran medida de la necesidad del cajista de justificar líneas ya que las mayúsculas ocupan doble espacio que las minúsculas.

En cuanto a las minúsculas, Zaragoza emplea exclusivamente y (1926) en clara oposición a Burgos, que la usa solo en una ocasión [a ${ }_{\text {vij }}$ : 4 frente 1542 signos tironianos. Toledo y Valencia utilizan ambos tipos si bien se contraponen en sus preferencias: Toledo 1343 frente a 169 signos tironianos, Valencia 262 y 1709 respectivamente.

La conclusión final a este respecto es que cada edición aplica sus propias preferencias o necesidades tipográficas más o menos definidas. 


\section{I- o Y- en posición inicial de palabra}

Se utiliza $i$ - inicial en las palabras compuestas por el prefijo negativo in- $y$ sus variantes $i m-, i^{20}$. Una singular excepción es ynocente, escrito así solamente una vez por las ediciones de Toledo $\left[d_{v i}{ }_{\mathrm{v}: 15}\right]$ y Burgos $\left[e_{i}{ }^{r: 10}\right]$ en el mismo pasaje. Hay que señalar que esta palabra la escriben Zaragoza $\left[\mathrm{d}_{\mathrm{j}}^{\mathrm{r}: 10}\right.$ y Valencia $\left[\mathrm{c}_{\mathrm{vj}}{ }^{\mathrm{v}: 23}\right]$ con doble $n$ : innocente siguiendo así la norma general; solamente cuando se pierde una $n$ difuminándose un tanto el prefijo in-, aparece esa única excepción, que, como veremos, obedece también a otra norma distinta. Por analogía, se utiliza igualmente $i$ - siempre que le sigue una - $n$ - o una - $m$-con tal que ambas formen sílaba ${ }^{21}$, lo que evidentemente no ocurre con la excepción anteriormente señalada de ynocente. Debemos sin embargo constatar que Burgos escribe una vez inocencia $\left[1_{\mathrm{ij}}^{\mathrm{v}: 1}\right.$ ) y otras dos innocencia $\left[\mathrm{b}_{\mathrm{iiii}}^{\mathrm{r}: 23}, \mathrm{~d}_{\mathrm{vi}}^{\mathrm{v}: 23}\right]$.

También se usa $i$ - en las palabras que comienzan con el prefijo im- siempre seguido de - $p$-, equivalente al in- anteriormente analizado y que Toledo conserva excepcionalmente en inportunidad $\left[\mathrm{k}_{\mathrm{ij}} \mathrm{v}: 9\right]$ en paralelo a importunidad $\left[\mathrm{a}_{\text {viij }} \mathrm{r}_{15}, \mathrm{~h}_{\mathrm{ij}}{ }_{\mathrm{v}}^{\mathrm{v} 27}, \mathrm{i}_{\mathrm{j}}^{\mathrm{v}: 28}\right]$. En el mismo pasaje en que Toledo ha escrito inportunidad, Burgos $\left[1_{v}{ }_{v}: 29\right]$, Zaragoza $\left[i_{v}{ }^{r: 25}\right]$ y Valencia $\left[i_{j}{ }_{j}: 22\right]$ prefieren iportunidad, con la línea de abreviación de la nasal sobre la $i$ - inicial.

Por el contrario, se utiliza $y$ - siempre que esta letra constituye por sí misma la sílaba inicial ${ }^{22}$,si bien existen algunas excepciones destacables que, en nuestra opinión, se deben a la colisión con la norma anterior.

Así encontramos ylicito en Burgos [ $\left.\mathrm{a}_{\mathrm{ij}}{ }_{\mathrm{r}}^{\mathrm{r}}: 18\right]$ frente a illicito en Toledo $\left[\mathrm{a}_{\mathrm{iiij}}{ }^{\mathrm{v}: 25}\right]$ e ilicito en Zaragoza $\left[a_{v}{ }^{v: 11}\right]$ y Valencia $\left[a_{v} r{ }^{39-40}\right]$. En este caso creemos que la norma alternativa opuesta es que la $i$-inicial es en realidad apócope del prefijo negativo in-, y lo mismo ocurre con yrrecuperables en Zaragoza $\left[h_{v}{ }_{v}^{v: 21}\right]$ frente a irrecuperable en Toledo $\left[k_{v j}^{r: 19}\right]$, Burgos $\left[m_{i j}{ }^{r: 28-29}\right]$, Zarago$z a\left[i_{\text {viij }}{ }^{v: 5}\right]$ y Valencia $\left[i_{\text {iiij }}\right.$ r:23] e irrecuperables en Valencia $\left[h_{j}{ }_{j}\right.$ r:25].

Asimismo, imitar dos veces en Toledo $\left[\mathrm{e}_{\mathrm{ij}}^{\mathrm{r}: 6}, \mathrm{~g}_{\mathrm{ij}}^{\mathrm{v}: 26}\right]$ y Burgos $\left[\mathrm{e}_{\mathrm{vi}}^{\mathrm{r}: 5}, \mathrm{~h}_{\mathrm{j}}^{\mathrm{r}: 24}{ }^{\mathrm{ili}}\right]$ y una en Valencia $\left[\mathrm{d}_{j}{ }^{v: 23}\right]$ frente a ymitar, dos en Zaragoza [ $\mathrm{d}_{\text {iiij }}^{\mathrm{r}: 1}, \mathrm{e}_{\mathrm{viij}}^{\mathrm{r}: 36}$ ] y otra en Valencia $\left[e_{v}{ }_{v}{ }^{2}: 22\right]$. Curiosamente Toledo [ $\left.{ }_{\mathrm{ij}}{ }_{\mathrm{v}}^{\mathrm{v}: 26}\right]$ pone y imitar, posible reflejo de una duda o titubeo por parte del cajista. También tenemos ymitauas en Toledo $\left[\mathrm{e}_{\mathrm{vij}}^{\mathrm{r}: 9-10}\right.$ ] pero imitauas en Burgos $\left[\mathrm{f}_{\mathrm{iiij}}^{\mathrm{r}: 7}\right]$ e imitaras en Valencia $\left[\mathrm{d}_{\mathrm{v}}{ }^{\mathrm{r}: 29}\right]$. Quizás el error provenga de ese comienzo por im- aunque en este caso ambas letras no formen sílaba ni se correspondan con el prefijo de negación.

También reseñable es la contraposición entre ynorancia, ynorante, ynorando, utilizadas solo en Toledo $\left[c_{j}{ }_{j}^{r: 10}, d_{v j}^{r: 15}, h_{v j}{ }_{v}^{v:}\right]$ una vez cada una, $e$

20.- Ejemplos: incierto, incomparable, incurable, impaciencia, impedir, inuisible, inmerito, incogitado, etc.

21.- Ejemplos: infernal, infierno, ingenio, inquina, inuierno, indicio, etc.

22.-Ejemplos: yda, ydo, yglesia, ygual, ymagen, ynes, ypócrita, yra, yran, yremos, ystoriales, yua, etc. 
ignorancia, ignorante, ignorando; en los primeros casos la $y$-forma sílaba por sí sola mientras que en el segundo la sílaba es ig-.

Finalmente, dos últimos casos: ignominiosos en Toledo [ $\left.\mathrm{e}_{\mathrm{iij}} \mathrm{r}: 26\right]$ frente a inominiosos en Burgos [ $\mathrm{e}_{\mathrm{vij}}^{\mathrm{v}: 7}$ ], Zaragoza [ $\mathrm{d}_{\mathrm{v}}^{\mathrm{r}: \mathrm{T}}$ ] y Valencia [ $\mathrm{d}_{\mathrm{ij}}^{\mathrm{ij}: 16}$ ] (comienzo in-), e ylustrantes en Toledo $\left[\mathrm{h}_{\text {viij }}^{\mathrm{v}: 21}\right]$ y Burgos $\left[\mathrm{k}_{\mathrm{ij}}^{\mathrm{r}: 10}\right]$ frente a illustrantes en Zaragoza $\left[g_{\text {iij }}\right.$ :10-11 $]$ y Valencia $\left[f_{\text {vij }}\right.$ r:18].

\section{I- o Y- en posición interior de palabra}

Podría afirmarse, con carácter general, que la -i- en el interior de la palabra va normalmente precedida de una consonante, incluyendo la $-h$ pese a su falta de sonido en la mayor parte de las ocasiones. No obstante, conviene mencionar dos casos en que la presencia de una - $u$ - precediendo a $-i$ - no contradice este enunciado ya que dicha - $u$ - carece del valor vocálico que lo haría falso:

1. La - $u$ - en interior de palabra no posee carácter de vocal, sino que equivale al fonema $/ v /$.

2. La $u$ de los dígrafos qu y gu no suena ante - $i$ - (ni tampoco ante -e-). Así en las sílabas qui y gui, como mezquino y conseguir, en las que el carácter mudo de la - $u$ - hace que el sonido anterior a la - $i$ - sea realmente el de las consonantes $q$ y $g$, velares oclusivas sorda y sonora respectivamente.

Apenas hay excepciones a la norma arriba enunciada de escribir - $i$ - detrás de un fonema consonántico, con centenares de testimonios. La única excepción que tiene consistencia es el uso de la - $i$ - detrás de - $u$-vocálica en el pretérito indefinido de los verbos ser e ir, sin que se note diferencia ortográfica entre las formas de ambos. A este respecto las divergencias son notables entre las cuatro ediciones. En efecto, Zaragoza utiliza el diptongo -ui- en nueve de diez ocasiones: fuiste (7), fuistes (1) y fuimos (1) ${ }^{23}$, mientras que Valencia sustituye la - $i$ - por -e-: fueste (8), fuestes (1) y fuemos $(1)^{24}$; Toledo emplea ambos diptongos -ui- (8) y -ue- $(2)^{25}$, así como Burgos: 6 y 4 respectivamente ${ }^{26}$.

23.- Zaragoza: fuiste [b iiij v:37, d vj r:35, d vj r:36, e ij v:35-36, e v v:20, e vj v:11, k j r:16], fuistes [d vj v:10-11], fuimos [i viij r:34]. Excepcionalmente, fuyste [d vij r:13].

24.- Valencia: fueste [b iij v:20, d iij v:29, d iij v:29, d iiij r:37, d vij v:33, e ij v:6, e iij r:27, i iiij v:29], fuestes [d iij v:41], fuemos [i iiij r:15].

25.- Toledo: fuiste [b vj v:11, e v r:4, e v r:4, f iij r:20, f viij r:24, k vij r:13], fuistes [e v r:19], fuimos [k vj r:8], fueste [e vj r:1, f vij r:15].

26.- Burgos: fuiste [f j v:1, f j v:2, g vj r:14, m iij v:2], fuistes [f j v:17], fuimos [m ij r:17], fueste [b vj v:7, f ij v:8, f viij v:21, g iiij v:26]. 
Reintegración, que aparece una sola vez en el texto, está con -i- en las cuatro ediciones ${ }^{27}$ y la razón parece lógica: si integración se escribe así dado su comienzo por in-, la anteposición del prefijo re- no es causa suficiente para cambiar la ortografía.

Conceptuamos las demás excepciones observadas de auténticas erratas o descuidos pues solo las encontramos una vez y en una sola edición y son: en Toledo poteneia $\left[b_{\mathrm{vi}} \mathrm{r}: 18\right]$; en Burgos posseido [ $g_{\mathrm{iiij}}^{\mathrm{v}: 9}$ ], retraimiento

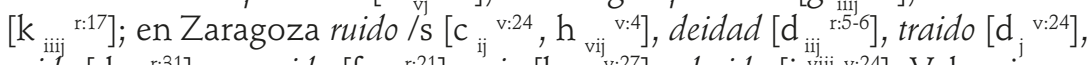
raido $\left[\mathrm{d}_{\mathrm{iij}}^{\mathrm{r}: 31}\right]$, proueida $\left[\mathrm{f}_{\mathrm{iiij}}^{\mathrm{r}: 21}\right]$, veia $\left[\mathrm{h}_{\mathrm{iiij}}^{\mathrm{v}}{ }_{\mathrm{v}}: 27\right.$ y huida $[\mathrm{i}$ viij $\mathrm{v}: 24]$. Valencia carece de estas anomalías.

Poteneia es una evidente errata por potencia, escrita así dos veces en la propia edición de Toledo, tres en Burgos y Valencia y dos en Zaragoza, que en otra ocasión escribe potentia.

Como contrapartida, las restantes palabras de que nos estamos ocupando se encuentran así en las demás ocasiones:

posseydo/s: una en Burgos y dos Toledo, Zaragoza y Valencia;

retraymiento: una en Burgos, dos en Toledo y tres en Zaragoza y Valencia; ruydo/s: seis en Zaragoza, cuatro en Toledo y Burgos y diez en Valencia, deydad: una en Toledo, Burgos y Valencia;

traydo: cuatro en Zaragoza y cinco en Toledo, Burgos y Valencia;

raydo: una en Toledo, Burgos y Valencia;

proueyda: una en Toledo, Burgos y Valencia;

veya: dos en Zaragoza, en Toledo y Valencia y tres en Burgos;

huyda: dos Zaragoza y otras dos Valencia más una fuyda, tres en Toledo y Burgos.

La ortotipografía de estas últimas palabras nos conduce a establecer otra norma seguida por los impresores: la $-y$-interior va siempre, salvo las escasas excepciones que también veremos, precedida de una vocal. Hemos registrado 3080 testimonios entre las cuatro ediciones. Las excepciones o erratas son realmente escasas, la mayoría en Toledo y en una sola ocasión: tyros $\left[\mathrm{a}_{\mathrm{vj}}^{\mathrm{r}: 20}\right]$, tynerias $\left[\mathrm{b}_{\mathrm{vj}}^{\mathrm{r}: 22}\right]$, cymera $\left[\mathrm{b}_{\mathrm{viij}}^{\mathrm{v}: 7}\right]$, mytad $\left[\mathrm{c}_{\mathrm{v}}^{\mathrm{v}: 14}\right]$, martyrios $\left[\mathrm{e}_{\mathrm{vj}}^{\mathrm{r}: 26}\right]$ y egypto $\left[\mathrm{h}_{\mathrm{v}}^{\mathrm{v}: 6}\right]$, y tres en Zaragoza: tynerias $\left[\mathrm{b}_{\mathrm{iiij}}^{\mathrm{v}: 24}\right]$, syendo $\left[\mathrm{c}_{\mathrm{ij}}{ }^{\mathrm{r}: 16}\right]$ y ryes $\left[\mathrm{g}_{\mathrm{j}}^{\mathrm{r}: 2}\right]$.

Procediendo de manera semejante al caso anterior, las contrapartidas a las excepciones citadas son: tiros en Burgos [ $\mathrm{a}_{\mathrm{iij}}^{\mathrm{v}: 25}$ ], Zaragoza [a $\mathrm{a}_{\mathrm{vi}}^{\mathrm{v}: 14}$ ] y Valencia $\left[\mathrm{a}_{\mathrm{vj}} \mathrm{r}: 33\right.$ ], y tiro en las cuatro ediciones: Toledo [ $\mathrm{e}_{\text {iiij }}$ v:26], Burgos $\left[\mathrm{f}_{\mathrm{j}} \mathrm{r}: 21\right]$, Zaragoza $\left[\mathrm{d}_{\mathrm{vj}} \mathrm{r}: 27\right.$ y Valencia $\left[\mathrm{d}_{\mathrm{iij}}^{\mathrm{v}: 21}{ }^{\mathrm{j}}\right.$; tenerias 14 testimonios entre las cuatro ediciones y cimera en Burgos $\left[b_{v i i j}{ }_{v}: 19\right]$, Zaragoza $\left[b_{v j}{ }_{\mathrm{v}}^{\mathrm{r}: 30}\right]$ y Valencia $\left[b_{i i i j}^{v: 37}\right]$, en la única vez que aparece esta palabra en el texto y lo mismo

27.- reintegración: Toledo [f viij v:15], Burgos [g vj v:9-10], Zaragoza [e v v:31], Valencia [e iij v:4]. 
ocurre con martirios en oposición a martyrios en Toledo $\left[\mathrm{e}_{\mathrm{vi}}^{\mathrm{r}: 26}\right.$. En cuanto a ryes, la forma con - $i$ - suma 19 entre las cuatro ediciones. Mas complicado es el caso de Egypto, para el que existen las siguientes lecturas: egypto en

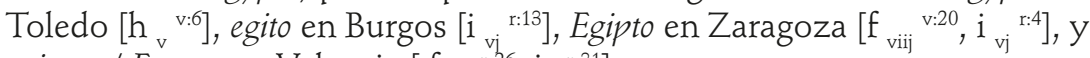
egiypto / Egypto en Valencia [ $\mathrm{f}_{\text {iiij }}^{\mathrm{v}: 26}, \mathrm{i}_{\mathrm{j}}{ }_{\mathrm{j}}^{\mathrm{v}: 31}$ ].

Cierto interés tiene el vocablo mitad, escrito así solamente una vez en Zaragoza [ $\left.\mathrm{c}_{\mathrm{ij}}^{\mathrm{r}: 13}\right]$, y meytad en Toledo $\left[\mathrm{d}_{\mathrm{vij}}^{\mathrm{r}: 20}\right]$, Burgos $\left[\mathrm{c}_{\mathrm{vij}}^{\mathrm{r}: 3}, \mathrm{e}_{\mathrm{ij}}^{\mathrm{v}: 10}\right]$, Zaragoza $\left[\mathrm{d}_{j}{ }^{\mathrm{v}: 35}\right.$ ] y Valencia $\left[\mathrm{c}_{\mathrm{vij}}{ }^{\mathrm{v}: 22}\right.$, formas ambas concordes con las normas ortotipográficas de colocar, respectivamente, $-i$ - detrás de consonante e $-y$ - detrás de vocal. Toledo presenta la lectura excepcional de mytad [ $\left.\mathrm{c}_{\mathrm{v}}{ }^{\mathrm{v}: 14}\right]$, que nos hace pensar en una simple errata debida a la omisión de la -e-.

Asimismo, en Zaragoza vemos syendo [ $\mathrm{c}_{\mathrm{ij}}{ }_{\mathrm{i}}^{\mathrm{r}: 16}$ contra seyendo en Valencia $\left[b_{\text {viij: }}{ }^{v: 12}\right]$ y siendo en Toledo $\left[c_{v}{ }^{v: 18}\right]$ y Burgos $\left[c_{v i j} \quad\right.$ r: $]$. Además esta es la forma que se repite 36 veces entre las cuatro ediciones.

Otro caso curioso en que se traslucen las normas que examinamos, $-i$ detrás de consonante e - $y$ - detrás de vocal, es el de la $3^{a}$ persona singular del imperfecto de ver, escrito via (11) y veya (9).

Por último y como curiosidad, donde Toledo $\left[h_{\text {viij }}\right.$ r:18 $]$ y Burgos $\left[k_{i} v: 3\right]$ ponen odio (lectura correcta), Zaragoza [ $\left.g_{\text {ij }}{ }^{v: 19}\right]$ y Valencia $\left[f_{v j}{ }_{v j}: 32\right]$ escriben oydo, evidente errata, pero las dos lecturas se ajustan a la norma.

\section{I- o Y- en posición final de palabra}

Mayoritariamente, la finalización en -i se produce en los monosílabos $m i$, ni, si, ti, cualquiera que sea su categoría gramatical. También se encuentra en palabras agudas en que la - $i$ va precedida de una consonante, con la aparente excepción de aqui, en la que, como ya se ha dicho, el carácter mudo de la $u$ en el dígrafo $q u$ - hace que la letra sonora anterior a la - $i$ sea la $q$. El otro conjunto de palabras acabadas en - $i$ está formado por verbos en primera persona del pretérito indefinido en que la letra anterior a la $-i$ es una consonante o una $-u$ - con valor de consonante, como por ejemplo serui (20). Solo hay dos sustantivos acabados en -i: nebli y marauedi, en que se da la circunstancia ya resaltada de tratarse de palabras agudas en que la $-i$ final va precedida de una consonante. La excepción más notable es fui, escrita así en Toledo (4), Burgos (4) y Zaragoza (2) 28 en coexistencia con fuy, también en Toledo (4), Burgos (5), Zaragoza (4) y Valencia $(2)^{29}$, lo que creemos se debe a la contraposición entre dos normas: la del final en $-i$ de las primeras personas de los pretéritos indefinidos y el rechazo a que la letra que precede a la $-i$ sea otra vocal. Retomaremos

28.- fui: Toledo [f viij r:28, g iij r:11, k v v:26, k vj v:12], Burgos [f iiij v:27, h j v:13, m ij r:j, $m$ ij v:26], Zaragoza [c vj r:26, g viij v:13].

29.- fuy: Toledo [d ij v:18, e vij v:23, k iij r:30, K iij v:2], Burgos [d v r:9, g vj r:18, 1 vj v:28, l vij r:7], Zaragoza [d viij r:36, c iiij v:3, e vj v:14, i ij v:27], Valencia [e j r:22, e v r:27]. 
esta cuestión tan pronto hayamos realizado el estudio del final en $-y$ de otras palabras. Valencia, consecuente con la sustitución de -i- por - e- en la conjugación del verbo ser que hemos visto en el apartado anterior, se aparta de estas normas escribiendo fue $\left[e_{\text {iii }}^{\mathrm{r}: 30}, \mathrm{i}_{\mathrm{iiii}}^{\mathrm{r}: 3}, \mathrm{i}_{\mathrm{iiij}}^{\mathrm{v}: 1}\right]$.

A lo largo de los textos hay en torno a 1450 palabras acabadas en $-y$ precedida de vocal, si bien no siempre se repiten en las cuatro ediciones, debido principalmente a las alteraciones producidas en el paso de Comedia a Tragicomedia. El 66\% se deben a tres palabras: ay, muy, oy. Es de resaltar que se trata en realidad de seis vocablos, ya que ay se utiliza como forma impersonal del verbo haber, como interjección y como adverbio de lugar, actual ahí. En cuanto a oy, puede ser tanto adverbio de tiempo como pretérito indefinido del verbo oyr.

El segundo grupo en importancia está constituido por las primeras personas del presente de indicativo soy (220), estoy (89), voy (82), doy (20).

Sustantivos se constatan cinco: rey (40), ley (23), menjuy (6), contray (4) y buey (4).

Tienen interés especial los pretéritos indefinidos, pues si, como vimos, cuando la penúltima letra era una consonante procedía que la última letra fuera $-i$, ahora nos encontramos con $-y$ precedida de vocal: fuy (16), cay (4), prouey (4).

La lista se completa con algunas palabras que consideramos especiales, como sey (20), imperativo de ser, la exclamación guay (14) y la onomatopeya de la risa $h y, h y, h y$ (19), escrita de esta forma en las cuatro ediciones pero que las Comedias convierten en $h i, h i, h i$ a partir del cuaderno $\mathrm{d}^{30}$. También se utilizan estas dos variantes en la apócope de hijo [de puta]: hi una vez en las Comedias ${ }^{31}$ e hy dos en Zaragoza [ $\left.\mathrm{e}_{\mathrm{vij}}^{\mathrm{r}: 17}, \mathrm{~h}_{\mathrm{viij}}^{\mathrm{r}: 27}\right]$, mientras que Valencia [ $\mathrm{e}_{\mathrm{iij}}^{\mathrm{v}: 26}, \mathrm{~h}_{\mathrm{iiij}}^{\mathrm{r}: 1}$ ] emplea una vez cada una.

Como conclusión, puede afirmarse sin género de dudas que en el empleo de $I$ o $Y$, tanto en el interior como en el final de palabra, el elemento determinante es que la letra precedente sea consonante o vocal respectivamente, sin que se tengan en cuenta las etimologías ni la categoría gramatical de las palabras.

\section{Usos de las grafías G, J, X, I, Y}

El uso de las grafías G, J, X está muy condicionado por la intensa evolución fonológica experimentada en el tránsito del español medieval al clásico. A este respecto dejó dicho el gramático Antonio de Torquemada en su Manual de escribientes: 
Estas tres letras traen en gran baraja y discordia la buena ortographía, porque con pareçer tan diferentes en sí, tienen tanta semejança en la pronunçiaçión, que muchas vezes se ponen la vna por la otra $[\ldots]^{32}$.

Pocos años después, López de Velasco aclarará que la $g$ ante las vocales a, o, $u$, suena como la gamma griega, mientras que delante de la e y de la $i$ tiene el mismo sonido que la $j$ (él la denomina «i larga») "sin diferencia ninguna en la voz». En cuanto a la $x$ afirma que los sonidos de la $g(+e, i)$ $y$ de la $x$ se han venido allegando de manera que apenas el oído percibe la diferencia que hay entre ellas, lo que origina dudas y errores en la pronunciación y por consiguiente en la escritura. También ve dificultad en las grafías $i$ e $y$ cuando actúan como "casi consonantes». ${ }^{33}$ Entendemos que López de Velasco apunta a una naturaleza fonético-articulatoria de las grafías consonánticas $j, g(+e, i), i, y, x$ cercana a lo que cabría describir aproximadamente como fonemas palatales (prepalatales o pospalatales) sonoros fricativos o africados ${ }^{34}$.

La estabilidad en la representación gráfica del fonema velar oclusivo sonoro mediante $g(+a, o, u$, consonante) en nuestros textos de referencia es total, no así la de los fonemas palatales.

En efecto, la inestabilidad en el uso de $j, g(+e, i), i, y, x$ se percibe en las cuatro ediciones de la Celestina que estudiamos, en las que existen alternancias de dichas grafías, bien debidas a diferencias de criterio entre unas ediciones y otras, o bien por vacilaciones y dudas dentro de cada una de ellas.

La alternancia entre estas letras se observa fundamentalmente en el interior de las palabras. En su comienzo hay pocas. Una es de carácter especial por tratarse de un nombre propio escrito «Gorge» en Toledo [ $\mathrm{d}_{\mathrm{v}}^{\mathrm{v}: 20}{ }$,

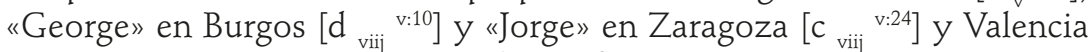
$\left[\mathrm{c}_{\mathrm{vi}}{ }_{\mathrm{r}}^{\mathrm{r}: 42}\right]$. En otras entran en juego las grafías $y-, j-, i$ -

Así donde Toledo $\left[\mathrm{d}_{\text {viij }}^{\mathrm{v}: 16}\right.$ y Burgos $\left[\mathrm{e}_{\mathrm{iiij}}^{\mathrm{r}: 2 \circ}\right.$ ] escriben ya mas y Zaragoza $\left[\mathrm{d}_{\mathrm{iij}} \mathrm{r}: 7\right]$ yamas, Valencia $\left[\mathrm{c}_{\text {vii. }}\right.$ v:20] prefiere jamas, y donde Toledo dice iusticia $\mathrm{y}$ iustos, las tres restantes eligen justicia y justos. ${ }^{35}$ La semejanza articulatoria entre la $j$-y la $i$ - se deduce también del hecho de que en ninguna de las cuatro ediciones existen palabras comenzadas por ji- (que fonéticamente podríamos asimilar a ii-), lo que refuerza López de Velasco diciendo que no hay ninguna palabra castellana que tenga tal comienzo.

32.- Transcripción y cita de Antonio Salvador Plans 1988: 361.

33.- Vid. López de Velasco, pp. 110-131, 160-165.

34.- Establecer la naturaleza fónico-articulatoria de tales sonidos en el siglo XVI es problemático pues los gramáticos de la época no precisan. Remitimos a José María Pozuelo Yvancos 1981: 57-61 para la cuestión.

35.- Toledo: iustos [d v r:14], iusticia [b j r:24, d v r:15, f j r:21]. Burgos: justos [d vij v:28], justicia [a vij r:26, a vij v:26, f vj v:10]. Zaragoza: justos [c viij r:24], justicia [a viij v:36, c viij r:25, e j v:7]. Valencia: justos [c vj r:6], justicia [a viij r:29, c vj r:6, d vj v:20] 
En lo que respecta al interior de las palabras y de acuerdo con ese diferente sonido de - $g$ - en función de las letras que la siguen, no encontramos palabra alguna en que - $g$ - alterne con $-j$ - ni con $-x$ - cuando la vocal siguiente es $a, o, u$, aunque debemos aclarar que sí existe alguna alternancia aparente; así, por ejemplo, encontramos en diversos lugares digo y dixo, pero no se trata de confusión de sonidos sino de establecer diferencias entre la primera persona del presente de indicativo y la tercera del pretérito indefinido.

Sin embargo, sí debemos señalar alternancias de $-j$ - con la $-y$ - y con la $-i$ - a consecuencia de sus similitudes fonéticas. Zaragoza [h$\left.{ }_{\mathrm{vi}}^{\mathrm{v}: 15}\right]$ y Valencia $\left[h_{i j}{ }^{r}: 28\right]$ escriben respectivamente trabajar y trabayar, y Toledo: a oyando $\left[\mathrm{d}_{\mathrm{vijj}}^{\mathrm{r}: \mathrm{i}}\right]$, ajamos $\left[g_{\mathrm{iij}}^{\mathrm{r}: 2}\right]$, obiecto [ $\left.\mathrm{a}_{\mathrm{v}}^{\mathrm{v}: 1}\right]$, ajuntamientos [b ij v:1], ajuda [b vij v:23], major $\left[\mathrm{C}_{\mathrm{iij}}{ }^{\mathrm{r}: 12}\right]$, mientras que Burgos, Zaragoza y Valencia prefieren a ojando o aojando, ayamos, objecto u objeto, ayuntamientos, ayuda y mayor ${ }^{36}$.

Hay que aclarar en relación con estas alternancias, que Zaragoza emplea el término ahoxar $\left[\mathrm{i}_{\mathrm{ij}}{ }^{\mathrm{v}: 34}\right]$, que instintivamente se podría relacionar con aojar de cuyo gerundio aojando acabamos de ocuparnos. Se daría así una alternancia rarísima que afectaría a -j-, -x- e -y-. Creemos no obstante que este ahoxar no tiene nada que ver con aojar, sino que son dos términos distintos, no solo por la - $h$-intercalada de este ahoxar (que cabría interpretar como errata o como recurso del cajista para justificar la línea o para "escusar el hiato» $)^{37}$, sino por el distinto significado que tienen ambos vocablos en sus respectivos contextos. En uno de ellos (Auto V) Celestina dice a Sempronio que lo que este necesita es:

vn arco para andar te de casa en casa tirando a paxaros y aojando paxaras a las ventanas: mochachas digo bouo $[\ldots]$ que no ay mejor alcahuete para ellas que vn arco [Zaragoza d ${ }_{\mathrm{ij}}^{\mathrm{v}: 3-6}$ ]

pareciendo claro que aquí aojando equivale a buscar con la vista muchachas en las ventanas para relacionarse con ellas.

En el otro caso (Auto XVIII) Centurio quiere que Traso el Coxo:

vaya dar vn repiquete de broquel a manera de lleuada para ahoxar vnos garçones [...] donde no conseguiran ningun daño mas de hazer los huyr y boluer se a dormir. [Zaragoza $\mathrm{i}_{\mathrm{ij}}$ v:33-36]

36.- a ojando o aojando: Burgos [e iij v:5], Zaragoza [d ii v:4], Valencia [c viij r:23-24] ayamos: Burgos [h j v:3], Zaragoza [e viij v:5-6], Valencia [e v r:19] objecto u objeto: Burgos [a iij r:1], Zaragoza [a vj r:7], Valencia [a v v:32] ayuntamientos: Burgos [b j v:15], Zaragoza [b j v:27], Valencia [b j r:6] ayuda: Burgos [b vij v:29], Zaragoza [b v v:27], Valencia [b iiij r:41] mayor: Burgos [c ij v:19], Zaragoza [b vij v:2], Valencia [b vi r:11]

37.- López de Velasco 1582:153. 
donde ahoxar, que la edición de Valencia sustituye por oxear, equivale a espantar, ahuyentar.

Existen alusiones a esta palabra en el Tesoro de la Lengua Castellana o Española de Covarrubias. Este incluye la entrada "ojeo», cuya definición es:

Término de caçadores [...] por la palabra repetida dellos de ox, que al parecer de algunos es del verbo latino exi.

Por su parte, López de Velasco, enunciando palabras con $\mathrm{X}$ «que no están por G», menciona:

oxe y oxear, de ox, voz para espantar las aues, que puede ser de oisseau frances, que es aue, y de alli oxear. (López de Velasco 250)

De entre las grafías cuyo uso analizamos, la - $g$ - es la única que se encuentra a principio de sílaba seguida de - $r$ - o de - $l$ - y a continuación una vocal, como en agradable, sangre, alegria, negro, etc. y regla, yglesia, negligente, gloria. Por el contrario, no existe ninguna $-j$ - seguida de consonante, lo que sí ocurre con la - $x$-, pero siempre al final de sílaba y tendiendo a convertirse en -s- en su evolución hacia el estado actual de la lengua, como en caxco, coxquillas y moxca; o hacia el valor actual de dicha letra, como en exceso o excellencia. También encontramos - $g$ - en fin de sílaba cuando la siguiente comienza por -n-, caso de digno, magnificola, ignorancia, magnificencia, magnes y magno; seguida de - $d$ - únicamente en Magdalena.

En cuanto a la secuencia interior - gu- no hay comentario que añadir si va seguida de una consonante o de las vocales $a, o$. Pero si va seguida de - $i$ o de -e, la articulación de la - $g$ - se asimila a la de ga, go. La - $u$ - no se pronuncia en la mayoría de las palabras: fatigues, ceguedad, etc.; mientras que en otros sí, caso de verguenza o antiguedad. La inexistencia de un signo gráfico, como la actual diéresis, pondría quizás en dudas a lectores poco versados, como quizá pasaría también con la falta de tildes. De esta alternancia del carácter mudo o sonoro de la - $u$ - puede ser testimonio lo que ocurre con el presente de subjuntivo del verbo seguir, si bien se trata de la sílaba -gua- que no debería ofrecer dudas en cuanto al carácter sonoro de la - $u-$, y además de que se trata de un verbo que lleva el dígrafo -gu-en su infinitivo. En las tres veces que aparecen dichas formas verbales en el texto nos encontramos con lo siguiente:

Toledo: siguas $\left[\mathrm{d}_{\text {viij }}\right.$ r:21], sigas $\left[\mathrm{i}_{\mathrm{vij}}^{\mathrm{v}: 10}\right]$, siganos $\left[\mathrm{k}_{\mathrm{j}}^{\mathrm{v}: 7-8}\right]$

Burgos: sigues $\left[\mathrm{e}_{\mathrm{iij}}^{\mathrm{v}: 21}\right]$, sigas $\left[1_{\mathrm{ij}} \mathrm{r}: 13\right]$, sigamos $\left[1_{\mathrm{iiij}} \mathrm{v}: 11\right]$

Zaragoza: siguas $\left[\mathrm{d}_{\mathrm{ij}} \mathrm{v}: 20\right]$, siguas $\left[\mathrm{g}_{\mathrm{viij}} \mathrm{r}: 36\right]$, sigua nos $\left[\mathrm{i}_{\mathrm{v}} \mathrm{r} \cdot 5\right]$

Valencia: siguas $\left[\mathrm{c}_{\text {viij }}{ }^{\mathrm{r}: 38}\right]$, siguas $\left[\mathrm{g}_{\mathrm{iiij}} \mathrm{r}: 18\right]$, siga nos $\left[\mathrm{h}_{\mathrm{viij}}{ }_{\mathrm{v}}: 21-22\right]$

Son muy pocas las palabras que incorporan la sílaba interior -ju- y ninguna de ellas ofrece alternancias con $-g u-$ ni con $-x u-$. En cuanto a esta 
solo aparece en luxuria (12) y enxuto (4) en las cuatro ediciones y axuar (2) en las dos Tragicomedias.

Sí dan lugar a numerosas alternancias algunas palabras que incluyen las sílabas -ge- y -je-como consecuencia probable de esa identidad de sonidos puesta de manifiesto por López de Velasco, aunque es frecuente que cada una de las cuatro ediciones se decante por una u otra grafía. Son ilustrativos los casos de mujerles, vejez/viejez, diversas formas del verbo envejecer y el adjetivo ajeno en ambos géneros y números, según puede observarse en la tabla $n^{\circ} 5$ :

Tabla ${ }^{\circ} 5$

\begin{tabular}{|l|c|c|c|c|c|}
\hline & Toledo & Burgos & Zaragoza & Valencia & Total \\
\hline muger/es & 50 & 46 & 14 & 72 & 182 \\
\hline mujer/es & 1 & 2 & 55 & 0 & 58 \\
\hline vegez & 9 & 9 & 2 & 0 & 20 \\
\hline$v($ i)ejez & 11 & 11 & 20 & 23 & 65 \\
\hline $\begin{array}{l}\text { Verbo } \\
\text { enuegescer }\end{array}$ & 1 & 1 & 1 & 1 & 4 \\
\hline $\begin{array}{l}\text { Verbo } \\
\text { enuejescer }\end{array}$ & 2 & 2 & 5 & 5 & 14 \\
\hline ageno/a/s & 13 & 13 & 1 & 17 & 44 \\
\hline ajeno/a/s & 0 & 0 & 19 & 3 & 22 \\
\hline
\end{tabular}

Otras palabras reflejan esa alternancia de forma muy excepcional. Así, de las 38 veces en que aparecen ligero y sus derivados, solo hallamos $l i$ jereza en Toledo [a $a_{v i i}{ }^{v 25}$ ], y de coger y derivados solo cojendo en Zaragoza $\left[e_{i}{ }^{v: 21}\right]$ y Valencia [d ${ }_{v i}^{v: 34}$. Únicamente aparecen una vez en la totalidad del texto pelligeros o peligeros, escrito con $-j$ - solo en Valencia $\left[b_{i} v: 37\right]$, estranjero también con - $j$ - solo en Valencia $\left[b_{\text {viij }}{ }^{\mathrm{r}: 38}\right]$ y coraje, que únicamente se encuentra en las Tragicomedias, escrito con-g-en Zaragoza $\left[\mathrm{e}_{\mathrm{viij}} \mathrm{v}: 24\right]$ y con $-j$ - en Valencia [ $\mathrm{e}_{\mathrm{v}}^{\mathrm{r}: 36}$.

Con superioridad de je: 78 mensaje y derivados por un solo mensages en Zaragoza [ $\mathrm{d}_{\mathrm{v}} \mathrm{r}: 33$ ] 46 linaje y derivados por un solo linage en Zaragoza $\left[c_{v i}{ }^{v: 22}\right.$, y 7 lisonjerola con $-j$ - por uno con $-g$ - en Burgos [i ${ }_{v i i j}$ r:10-11 $]$.

Aparece solo una vez en el texto hereje, escrito con -g-en Zaragoza $\left[a_{v j}{ }^{v: 2}\right]$ y con $-j$ - en Toledo $\left[a_{v j}\right.$ r:6], Burgos $\left[a_{i i j}^{v: 10}\right]$ y Valencia $\left[a_{v j}\right.$ : $\left.: 21\right]$.

\section{Conclusión: normas de escritura}

Habiendo estudiado los textos de las cuatro ediciones en conjunto desde el punto de vista de la cuantificación, catalogación y valoración de los usos de $h, i, y, g, j, x$ en miles de vocablos, enunciamos las siguientes nor- 
mas de escritura generales (en las que no faltan las excepciones constatadas quizá debidas a erratas, descuidos o contaminación entre normas) que deducimos para cada una de estas grafías, encasillándolas en tres grupos según hicimos en el anterior artículo del que, como hemos señalado, este es continuación:

A) De carácter ortográfico:

- Unanimidad casi absoluta en el empleo de la $h$ - inicial en más de mil palabras en el conjunto de las cuatro ediciones.

- El verbo (h)auer destaca por la inestabilidad de la $h$-, con preferencia por omitirla en la flexión de todos los tiempos menos en la del presente de indicativo, que la llevan mayoritariamente las cuatro ediciones. La mayor frecuencia de $h$ - en las demás formas la presenta la edición burgalesa, en contraste con la escasez de la valenciana.

- Predominio de $h$-inicial en oposición a f-en una proporción que alcanza el 90\%. La edición de Zaragoza destierra prácticamente la f-.

- Uso de $i$ - inicial en las palabras compuestas por el prefijo de negación in-, im-, $i-$, y por analogía, en los demás vocablos que comienzan por $i$ - seguida de $-n$ - o de $-m$ - con tal de que ambas letras formen sílaba.

- Empleo de -i- en interior de palabra cuando va precedida de consonante o de - $u$ - carente de valor vocálico. También es constante el uso de - $i$ final de palabra en dichas condiciones.

- Empleo de $y$-vocal al principio de palabra cuando esta letra forma sílaba por sí misma.

- Empleo de y con valor vocálico en interior y en fin de palabra cuando va precedida de vocal.

- Uso unánime de $g$ ante $a, o, u, r, l, n$, sea cual sea el lugar que ocupe en la palabra.

- Alternancia en el uso de $j(+e)$ y de $g(+e)$.

- Los escasos y alternantes datos referentes a la representación gráfica de los fonemas cercanos al punto de articulación palatal en evolución fonológica ( $x, j$ e $y, i$ consonantes) muestran la vacilación característica de la época.

B) De carácter tipográfico:

- Presencia de $h$ - inicial ante el diptongo -ue-con el fin de que la $u$ no pase tipográficamente a $v$ - ocasionando el confusionismo consiguiente. 
- Preferencia por $h$ - inicial en el presente de indicativo del verbo (h)auer, quizá por motivación estética, y por su ausencia en los demás tiempos.

- Uso de ay con los significados de adverbio de lugar, exclamación e impersonal del verbo (h)auer.

- Conversión de hi-en $y$-inicial en las palabras en que se ha omitido la $h$ -

- En la representación de la conjunción copulativa, cada edición aplica sus propias preferencias o necesidades tipográficas. La edición de Zaragoza únicamente escribe $y$, mientras que Burgos elige el signo tironiano para las minúsculas y E para las mayúsculas. Toledo y Valencia alternan las tres formas.

C) De carácter estético:

- Sugerimos que el especial predominio $(92,87 \%)$ de la $h$-inicial en el presente de indicativo del verbo (h)auer pudiera deberse al deseo de aumentar la imagen visual de los monosílabos e, as, a, an con el fin de que no perdieran identidad entre las palabras contiguas, separadas a veces por espacios tan reducidos como imponía el ajuste de líneas en las compactas y densas páginas de los impresos en letra gótica. 


\section{Bibliografía citada}

Berndt-Kelley, Erna (1977), "Algunas observaciones sobre la edición de Zaragoza de 1507 de la Tragicomedia de Calisto y Melibea», en Manuel Criado de Val (coord.), "La Celestina" y su contorno social. Actas del I Congreso Internacional sobre la "Celestina", Barcelona, Hispam, pp. 7-30.

BotTA, Patrizia y Víctor Infantes (1999), "Nuevas bibliográficas de la Tragicomedia de Calisto y Melibea (Zaragoza, Jorge Coci, 1507)", Revista de Literatura Medieval XI, pp. 179-208.

CANet Vallés, José Luis (2005), «Los correctores de imprenta (y/o componedores) como configuradores de las normas de escritura (un caso entre Valencia-Sevilla en la primera mitad del siglo XVI)", en Filologia dei testi a stampa (Area Iberica), ed. a cura di Patrizia Botta, Modena, Mucchi Editore, pp. 369-380.

- (ed.) (2011), Comedia de Calisto y Melibea. Edición crítica, introducción y notas, Valencia, PUV, col. Parnaseo.

- (2014), "De nuevo sobre cajistas y correctores de las ediciones de la Comedia de Calisto y Melibea», Epos, XXX, pp. 113-126.

Comedia de Calisto y Melibea (1500), Toledo, Pedro Hagenbach. Facsímil de la edición de Toledo 1500, ed. Daniel Poyán Díaz,Cologny-Ginebra, Biblioteca Bodmeriana, 1961.

Comedia de Calisto y Melibea [1499-1502?], Burgos, Fadrique de Basilea. Edición facsimilar de Archer M. Huntington, New York, Hispanic Society of America, 1909 (Reimpresa en 1970)

Comedia de Calisto y Melibea [1499-1502?], Burgos, Fadrique de Basilea. Edición facsimilar de Emilio de Miguel Martínez, Salamanca, Juntade Castilla y León-Caja Duero, Ediciones Universidad de Salamanca, 1999, 2 vols.

Corfis, Ivy A. and John O'Neill (General Editors) (1997), Early Celestina. Electronic Texts and Concordances, Hispanic Seminary of Medieval Studies, Madison, CD-ROM.

García de LuCAS, César (2008), "Más notas sobre la lengua de La Celestina», en Rica Amram (ed.), Autour de La Celestina, París, Indigo, pp. 163-174.

López de Velasco, Juan (1582), Orthographia y Pronunciacion Castellana, Burgos.

Marciales, Miguel (ed.) (1985), Fernando de Rojas, Celestina. Tragicomedia de Calisto y Melibea, Urbana - Chicago, University of Illinois Press (Illinois Medieval Monographs, I), 2 vols. 
Moralejo Álvarez, María Remedios (2018), "Una Celestina desconocida», en La Celestina de Fernando de Rojas sale de nuevo a la luz en facsimil con una introducción de $M^{a}$ Remedios Moralejo Álvarez. [Edición a expensas y al cuidado de Alfonso Fernández González. Bibliófilo].- Zaragoza-Sabadell. Establecimiento tipográfico Francesc Fusté Serena. 2018.

NebRIJA, Antonio (1492), Gramática de la lengua castellana, Salamanca. Estudio y edición de Antonio Quilis, Madrid, Editorial Centro de Estudios Ramón Areces, 1990.

Pozuelo Yvancos, José María (1981), López de Velasco en la teoría gramatical del siglo XVI, Murcia, Universidad de Murcia.

PRIETO de LA IgLeSIA, Remedios (2014), «Erratas y corrector de la impresión: Alonso de Proaza y Celestina», Celestinesca 38, pp. 113-124.

- (2015), "Constricciones y libertades ortográficas de los impresores en cuatro ediciones tempranas de Celestina: Toledo 1500, Burgos 14991502 (?), Zaragoza 1507 y Valencia 1514», Titivillus 1, pp. 237-249.

- y Sánchez Sánchez-Serrano, Antonio (2016), "Posibles razones por las que la Celestina fue considerada anónima durante los siglos XVI-XvIII y creación de Rojas a partir del XIX», Celestinesca 40, pp. 135-158.

Salvador Plans, Antonio (1988), «Ideas lingüísticas de Antonio Torquemada", Anuario de estudios filológicos 11, pp. 349-369.

Sánchez SÁnchez-Serrano, Antonio (2014), "Las abreviaturas en cuatro ediciones tempranas de la Celestina: Toledo 1500, Burgos 1499-1502 (?), Zaragoza 1507 y Valencia 1514. Catalogación, cuantificación y consecuencias editoriales», Celestinesca 38, pp. 125-154.

Sebastián Mediavilla, Fidel (2003), "Las primeras ediciones de La Celestina y su puntuación", Boletín de la Real Academia Española, tomo LXXXIII, cuaderno CCLXXXVII, pp. 113-135.

Snow, Joseph Thomas (1999-2000), «Fernando de Rojas, ;autor de Celestina?», en Studia Hispanica Medievalia V. Actas de las VI Jornadas Internacionales de Literatura Española Medieval, eds. A. Liotta \& S. Lupi, Letras 40-41, Buenos Aires, Universidad Católica de Buenos Aires, pp. 152-157.

- (2001), "Los estudios celestinescos 1999-2099», en La Celestina. V Centenario (1499-1999), Actas del Congreso Internacional Salamanca, Talavera de la Reina, Toledo, La Puebla de Montalbán, 27 de septiembre a 1 de octubre de 1999, eds. Felipe B. Pedraza Jiménez, Rafael González Cañal y Gema Gómez Rubio, Cuenca, Universidad de Castilla-La Mancha, pp. 121-130.

SNow, Joseph Thomas (2005-2006) «La problemática autoría de Celestina», Íncipit 25-26, pp. 537-561.

TRAGiComedia DE CALISTO y MeLIBEA (1507), Zaragoza, Jorge Coci. Edición facsimilar y estudios coordinados por Julián Martín Abad, en Un volumen facticio de raros post-incunables españoles, Toledo, Antonio Pareja Editor, 1999, 2 vols. 
Tragicomedia de CaListo y Melibea (1514), Valencia, Juan Joffre (Biblioteca Nacional de España: R/4870. Reproducción facsimilar en Biblioteca Virtual Miguel de Cervantes).

TRaGicomedia DE CALISTO y Melibea (1514). Edición facsimilar, Madrid, Espasa-Calpe, 1975.

TRAGiCOMEDIA DE CALISTO y Melibea (1514). Edición facsimilar, Barcelona, Círculo del Bibliófilo, 1977.

Tragicomedia de Calisto y Melibea (Valencia, Juan Joffre, 1514). Estudios y edición paleográfica y facsimilar, dirigida por Nicasio Salvador Miguel y Santiago López-Ríos, Valencia, Institució Alfons el Magnànim, 1999, 2 vols. 